\title{
Business Process Adaptation Based on Process Variants*
}

\author{
Hong Xia $\mathrm{Li}^{1,2}$, Yu Yue Du ${ }^{1}$ \\ 1. College of Information Science and Engineering, \\ Shandong University of Science and Technology, Qingdao 266590, China \\ 2. College of Science and Information, Qingdao Agricultural University \\ Qingdao, 266109, China \\ lovelymelody@163.com,yydu001@163.com
}

\begin{abstract}
An extensional method for existing business processes is proposed to adapt new environments based on process variants in this paper. The core processes describing maximal commonalities of process variants are introduced according to inheritance relation. Then, extensional business processes are defined based on the core processes. The algorithms of the core processes and extensional business are given and proved. And the soundness of two kinds of processes is justified. An example shows that extensional business processes can adapt to the new environment better than the original process.
\end{abstract}

Keywords: commonality; inheritance relation; core process; extensional business process

\section{Introduction}

Business Process Management (BPM) combines the knowledge from information technology and the one from management sciences, and is applied to operational business processes [1]. Many enterprises have many business processes, and the same business process may exist in the form of multiple variants [2]. New business processes can be constructed easily from process variants, but variant management is difficult.

Many methods are proposed to regulate and reuse similar business processes, i.e. process variants, such as a reference process model [3] and a configurable process model [4-5]. A reference process model tries to capture recurrent processes to gain a standard reference model repository from the process variants, and a specific process model can be realized manually for individualization. A configurable process model represents multiple business process model variants in an integrated manner. It can be built by recognizing [6-7] and merging [8] similar business processes. A specific process model is decided at the build time or run time [9].

The above models are based on the hypothesis that an individual business process is an alternative of the integrated business process model. In fact, it is difficult to get a specific process directly from existing process variants, and it is often necessary to adapt a business process to specific environment like specific law or company culture [9]. In this paper, core processes are proposed to denote maximal commonalities of process variants, and a business process can be extended based on core processes to meet specific environment requirement.

In term of modeling language, there exist many different notations for process models, such as labeled transition systems, Event-driven Process Chains (EPC), Business Process Modeling Notation (BPMN), Petri nets, and workflow nets (WF-nets). Petri nets have been extensively applied to the formal verification of business process models [10-14].

* Corresponding author: Yu Yue Du. Email: yydu001@163.com 
WF-nets [11] allow for a clear definition with initial and final states to describe the process start and end. Therefore, we choose WF-nets to model the process in this paper.

The rest of the paper is structured as follows. Section 2 introduces preliminaries. Section 3 discusses the concepts and algorithms of the core process (CP) and extensional business process (EBP). Then, the algorithms and the soundness of these processes are proved. In section 4, declare tax processes are introduced to illustrate the extensional method of business processes. The related works and conclusions are shown in Sections 5 and 6 respectively.

\section{Preliminaries}

Some concepts are introduced in this section. The definition of labeled P/T-nets [15] is reviewed firstly. Then behavioral equivalence and inheritance relation are presented.

Definition 1 (Labeled P/T-net) A labeled P/T-net is a class of Petri nets, described as a tuple $L N=(P, T, F, L)$, where

(1) $P$ is a finite set of places;

(2) $T$ is a finite set of transitions, such that $P \cup T \neq \varnothing, P \cap T=\varnothing$;

(3) $F \subseteq(P \times T) \cup(T \times P)$ is a set of directed arcs of $P$ and $T$, called the flow relation;

(4) $\operatorname{dom}(F) \cup \operatorname{cod}(F)=P \cup T$ where

(5) $\operatorname{dom}(F)=\{x \in P \cup T \mid \exists y \in P \cup T:(x, y) \in F\}$

(6) $\operatorname{cod}(F)=\{y \in P \cup T \mid \exists x \in P \cup T:(x, y) \in F\}$ and

(7) $L: T \rightarrow A \cup \tau$ is a labeling function, and $A$ is a set of activities and $\tau$ denotes an invisible activity.

Definition 2 (Pre-set and Post-set) Let $L N$ be a labeled P/T-net, $x \in P \cup T$ be an element of $L N$, then

- $x=\{y \mid y \in P \cup T \wedge(y, x) \in F\}$ is called the pre-set of $x$;

$x^{\bullet}=\{y \mid y \in P \cup T \wedge(x, y) \in F\}$ is called the post-set of $x$.

A class of Petri nets, namely workflow nets, is specifically designed for business process modelling. Each workflow net has a single starting point and an ending point, which represents the creation and completion of a business process. Each process instance progresses independently through a series of activities until completion. The definition of labeled WF-net is described in the following, and a silent transition being not observed is labelled by the symbol $\tau$.

Definition 3 (Labeled WF-net) Let $L N=(P, T, F, L)$ be a labeled P/T-net. And $t^{\prime}$ is not in $P \cup T$ and labelled by $\tau$. Then $N$ is a labeled Workflow net (labeled WF-net) iff:

(1) Process creation: it has an only source place $i$ and ${ }^{\circ} i=\varnothing$;

(2) Process completion: it has an only sink place $o$ and $o^{\circ}=\varnothing$; and

(3) Connectedness: $N^{\prime}=\left(P, T \cup\left\{t^{\prime}\right\}, F \cup\left\{\left(o, t^{\prime}\right),\left(t^{\prime}, i\right)\right\}, L \cup\left\{\left(t^{\prime}, \tau\right)\right\}\right)$ is strongly connected.

Definition 4 (Firing rule) Let $N=(P, T, F, L)$ be a labeled WF-net with source place $i$ and sink place $o$ :

(1) $M: P \rightarrow\{0,1,2, \ldots\}$ is a marking of $N$;

(2) $M_{i}$ is the initial marking of $N$ with one token in i, i.e. $M_{i}=[i]$;

(3) $M_{o}$ is the final marking of $N$ with one token in o, i.e. $M_{o}=[o]$;

(4) For all $p, p \in{ }^{*} t$, if $M(p) \geq 1$, then $t$ is called enabled under $M$, denoted as $M[t>$. If $M$ [ $t>$ and $t$ is fired, then a new marking $M^{\prime}$ is generated, denoted by $M\left[t>M^{\prime}\right.$, where 


$$
M^{\prime}(p)=\left\{\begin{array}{cl}
M(p)+1 & \text { if } p \in t^{\bullet}-{ }^{\bullet} t \\
M(p)-1 & \text { if } p \in \bullet^{\bullet} t-t^{\bullet} \\
M(p) & \text { el se }
\end{array}\right.
$$

The set of all labeled WF-nets is denoted $\Phi(N)$.

To distinguish the descriptive power of different labeled WF-nets, inheritance relation and behavioral equivalence are introduced. Branching bisimilarity [16] is chosen as the standard equivalence relation due to not observed transitions. Branching bisimilarity is defined as follows.

Definition 5 (branching bisimilarity) Let $N=(P, T, F, L)$ be a labeled WF-net, and a binary relation $R \subseteq(\Phi(N) \times \Phi(N))$ is called a branching bisimulation iff, for any $p, p^{\prime}, q$, $q^{\prime} \in \Phi(N)$ and $a \in A \cup\{\tau\}:$

(1) $p R q \wedge p\left[a>p^{\prime} \Rightarrow \exists q^{\prime}:\left(q\left[a>q^{\prime}\right) \wedge\left(p^{\prime} R q^{\prime}\right)\right.\right.$;

(2) $p R q \wedge q\left[a>q^{\prime} \Rightarrow \exists p^{\prime}:\left(p\left[a>p^{\prime}\right) \wedge\left(p^{\prime} R q^{\prime}\right)\right.\right.$; and

(3) $p R q \Rightarrow p$ can properly complete iff $q$ can properly complete.

Two labeled WF-nets are called branching bisimilar, denoted $p \sim q$, if and only if there is a branching bisimulation $R$ such that $p R q$. Branching bisimilarity is an equivalence relation on $\Phi(N)$, i.e. $\sim$ is reflexive, symmetric, and transitive [16].

The behavioral equivalence between labeled WF-nets based on branching bisimilarity can be defined as follows.

Definition 6 (behavioral equivalence) Let $N_{1}$ and $N_{2}$ be labeled WF-nets, then $N_{1}$ and $N_{2}$ satisfy behavioral equivalence iff $\left(N_{1},\left[i_{1}\right]\right) \sim\left(N_{2},\left[i_{2}\right]\right)$.

Hiding and blocking operation are introduced to define inheritance relation. Hiding a transition refers to skipping its execution while it is fired, without affecting the rest of the process. Blocking a transition implies to inhibit it in the process model. Blocked transitions cannot forward processes and all the subsequent transitions will never be executed unless they can be enabled via other paths [5].

Definition 7 (hiding operation) Let $N=\left(P, T, F, L_{0}\right)$ be a labeled WF-net, for any $I \subseteq A$ $\left(L_{0}: T \rightarrow A \cup \tau\right.$ ), hiding operation $\tau_{I}$ can be described as follows.

$\tau_{I}(N)=\left(P, T, F, L_{1}\right)$, where

(1) If $L_{0}(t) \in I$, then $L_{1}(t)=\tau$; and

(2) If $L_{0}(t) \notin I$, then $L_{1}(t)=L_{0}(t)$.

Definition 8 (blocking operation) Let $N=\left(P_{0}, T_{0}, F_{0}, L_{0}\right)$ be a labeled WF-net, for any $H \subseteq A_{0}\left(L_{0}: T_{0} \rightarrow A_{0} \cup \tau\right)$, blocking operation $\partial_{H}$ can be described as follows.

$\partial_{H}(N)=\left(P_{1}, T_{1}, F_{1}, L_{1}\right)$, where

(1) $T_{1}=\left\{t \mid t \in T_{0} \wedge L_{0}(t) \notin H\right\}$

(2) $P_{1} \subseteq P_{0}$;

(2) $F_{1}=F_{0} \cap\left(\left(P_{1} \times T_{1}\right) \cup\left(T_{1} \times P_{1}\right)\right)$; and

(3) $L_{1}=L_{0} \cap\left(T_{1} \times A\right)$

Inheritance relation is defined based on the hiding operation, blocking operation and behavioral equivalence. A labeled WF-net inherits from another labeled WF-net if and only if they satisfy behavioral equivalence by blocking and/or hiding its one or more transitions.

Definition 9 (inheritance relation) Let $N_{1}$ and $N_{2}$ be labeled WF-nets. If $\exists I$ and $H$, such that $\left(\tau_{\rho^{\circ}} \partial_{H}\left(N_{1}\right),[i]\right) \sim\left(N_{2},[i]\right)$, where $I, H \subseteq A_{1}$ and $I \cap H=\varnothing$, then $N_{1}$ and $N_{2}$ are called satisfy inheritance relation. Meanwhile, $N_{1}$ is a subclass of $N_{2}$, denoted as $N_{1} \leq N_{2}$, and $N_{2}$ is a superclass of $N_{1}$.

\section{Adaptation of Business Processes based on the Core Process}


Sometimes, it is necessary to adapt business processes to a new environment, for example, the enterprise moves to another country. The existing process variants in the new country are helpful to the adaption of business processes. Firstly, a CP is proposed to describe the maximal commonalities of business process variants in the new environment. Then, an EBP is defined to describe the process adaption based on the CP. The EBP keeps the characteristic of original business process and adapt to the new environment well. The concepts and algorithms of the CP and EBP are given, and the soundness of algorithms and processes are verified in this section.

\subsection{Construct CP}

A CP, which describes the maximal commonalities of business process variants, should capture maximum activities and their relations of process variants. And the $\mathrm{CP}$ can be defined as follows.

Definition 10 (Core Process, $\boldsymbol{C P}$ ) Let $N_{0}=\left(P_{0}, T_{0}, F_{0}, L_{0}\right)$ be a labeled WF-nets with only sequential structure. Let $N_{i}=\left(P_{i}, T_{i}, F_{i}, L_{i}\right)(1 \leq i \leq \mathrm{n})$ be labeled WF-nets of process variants. Then a labeled WF-net $N=(P, T, F, L)$ is a CP of $N_{i}$ iff:

(1) $N_{i} \leq N(1 \leq i \leq \mathrm{n})$, and

(2) If $\exists N_{0}$ such that $N_{i} \leq N_{0}(1 \leq i \leq \mathrm{n})$, then $N \leq N_{0}$.

Note that a core process is a superclass of process variants. Furthermore, for any a superclass of process variants, a core process is a subclass of the superclass, i.e. a core process embodies the maximal commonalities of process variants.

The set of CPs of process variants is denoted CPset.

The execution of a process instance should satisfy proper creation and proper completion without deadlock. A labeled WF-net fulfilling these requirements is sound. Formally:

Definition 11 (sound) Let $N=(P, T, F, L)$ be a labeled WF-net. $N$ is sound iff:

(1) Proper completion: for any marking $M \in[N,[i]>, o \in M$ implies $M=[o]$;

(2) Option to complete: for any marking $M \in[N,[i]>,[o] \in[N, M>$; and

(3) No dead transitions: $(N,[i])$ contains no dead transitions.

A trace of a labeled WF-net is put forth to describe a process activity sequence. And CPset of process variants can be acquired according to the traces.

Definition 12 (trace) Let $N$ be a labeled WF-net, and $\sigma=[i] t_{1} M_{1} t_{2} M_{2} t_{3} \ldots M_{m-1} t_{m}[o]$ be a finite occurrence sequence. And the corresponding activity sequence of the occurrence sequence $t_{N}=L\left(t_{1}\right) L\left(t_{2}\right) L\left(t_{3}\right) \ldots L\left(t_{m}\right)$ is a trace of $N$. And $L\left(t_{i}\right) \quad(1 \leq i \leq m)$ is the small component unit of $t_{N}, L\left(t_{i}\right) \in t_{N}(1 \leq i \leq m)$.

The trace set of a labeled WF-net $N$ is denoted $F_{N}$.

Let $N_{1}$ and $N_{2}$ be labeled WF-nets, then each trace of $N_{1}$ and $N_{2}$ can be expressed as a string of $L(t)$. Thus, many string functions can be applied in traces, such as string concatenation (strcat), substring, strlength (strlen), longest common substring (lcs). The result of one string strcat $\tau$ is the string itself. For the trace sets $F_{N_{1}}$ of $N_{1}$ and $F_{N_{2}}$ of $N_{2}$, the longest common substring set of $N_{1}$ and $N_{2}$ is denoted $\operatorname{LCS}\left(F_{N_{1}}, F_{N_{2}}\right)$.

Let $N_{1}, N_{2}$ be process variants of a common process. The CPset of $N_{1}$ and $N_{2}$ can be constructed in the following.

\footnotetext{
Algorithm 1 Construct CPset

Input: Labeled WF-nets $N_{1}=\left(P_{1}, T_{1}, F_{1}, L_{1}\right)$ and $N_{2}=\left(P_{2}, T_{2}, F_{2}, L_{2}\right)$

Output: $\Phi$
} 


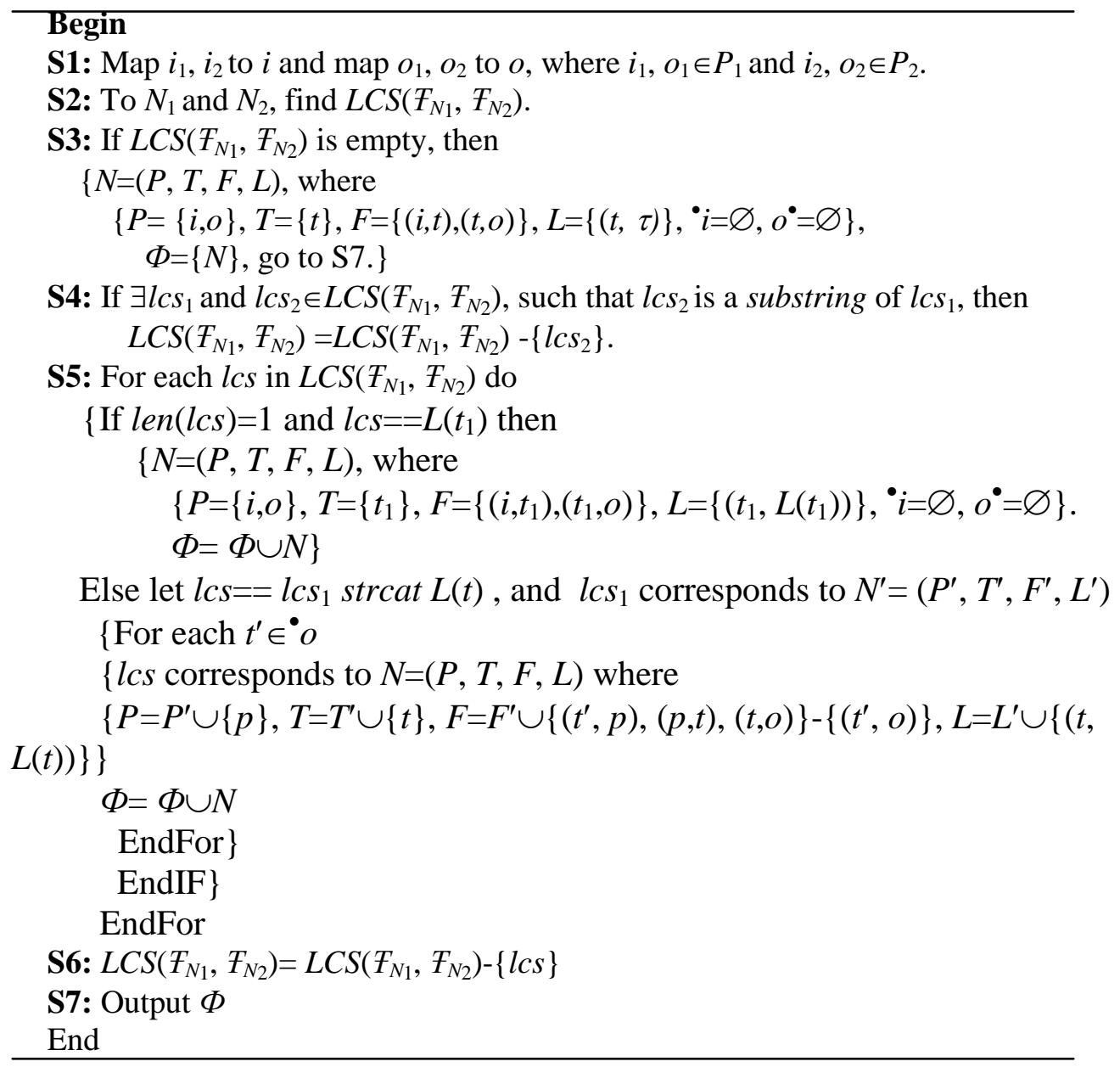

Figure 1. The CPset Construction Algorithm

In Algorithm 1, $\operatorname{LCS}\left(F_{N} 1, F_{N} 2\right)$ is used to construct $\Phi$. So, each element in $\Phi$ captures the maximal commonalities of $N_{1}$ and $N_{2}$. Following conclusion proves that what we got by Algorithm 1 is the CPset of $N_{1}$ and $N_{2}$.

Theorem 1 Let $N_{1}=\left(P_{1}, T_{1}, F_{1}, L_{1}\right)$ and $N_{2}=\left(P_{2}, T_{2}, F_{2}, L_{2}\right)$ be labeled WF-nets of a business process. $N_{0}=\left(P_{0}, T_{0}, F_{0}, L_{0}\right)$ is a labeled WF-net with only sequential structure. Let $N=(P, T, F, L)$ be an element of $\Phi$ by Algorithm 1, then $N$ is a CP of $N_{1}$ and $N_{2}$.

Proof. (1) Since $N$ just has the sequential structure (Algorithm 1), i.e. $N$ has an only occurrence sequence $\sigma=[i] t_{1} M_{1} t_{2} M_{2} t_{3} \ldots M_{m-1} t_{m}[o]$, obviously, $N$ has an only source place $i$ and sink place $o$ and ${ }^{\circ} i=\varnothing, o^{\circ}=\varnothing$. Meanwhile, Let $N^{\prime}=\left(P, T \cup\left\{t^{\prime}\right\}, F \cup\left\{\left(o, t^{\prime}\right),\left(t^{\prime}, i\right)\right\}\right.$, $\left.L \cup\left\{\left(t^{\prime}, \tau\right)\right\}\right)$ where $t^{\prime}$ is not in $P \cup T$, then $N^{\prime}$ is strongly connected. So, $N$ is a labeled WFnet.

(2) In $N$, for $\forall t \in T$ such that $L(t) \in A$, there must exist a trace $t_{N_{1}}$ of $N_{1}$ and a trace $t_{N_{2}}$ of $N_{2}$, such that $L(t) \in t_{N_{1}}$ and $L(t) \in t_{N_{2}}$.

Let $F_{N_{1}}$ be the trace set of $N_{1}$. For $N_{1}$, let $I=\left\{L_{1}(t) \mid L_{1}(t) \in t_{N_{1}} \wedge L_{1}(t) \notin A\right\}, l c s_{1} \in F_{N_{1}}\left\{\left\{t_{N_{1}}\right\}\right.$ and $H=\left\{L_{1}(t) \mid L_{1}(t) \in l c s_{1} \wedge L_{1}(t) \notin A\right\}$, then $\left(\tau_{I} \circ \partial_{H}\left(N_{1}\right),[i]\right) \sim(N,[i])$, i.e. $N_{1} \leq N$.

$N_{2} \leq N$ can be proved similarly.

(3) If $\exists N^{\prime}=\left(P^{\prime}, T^{\prime}, F^{\prime}, L^{\prime}\right)$ such that $N_{1} \leq N^{\prime}, \quad N_{2} \leq N^{\prime}$. For $\forall t^{\prime} \in T^{\prime}$ such that $L\left(t^{\prime}\right) \in A^{\prime}$, there are $L\left(t^{\prime}\right) \in A_{1}$ and $L\left(t^{\prime}\right) \in A_{2}$, i.e. $\exists l c s_{2} \in L C S\left(F_{N_{1}}, \quad F_{N_{2}}\right)$ such that $L\left(t^{\prime}\right) \in l c s_{2}$. Let $I=\left\{L(t) \mid L(t) \in l c s_{2} \wedge L(t) \notin A^{\prime}\right\},\left(\tau_{I}(N),[i]\right) \sim\left(N^{\prime},[i]\right)$, i.e. $N \leq N^{\prime}$.

Therefore, $N$ is a CP of $N_{1}$ and $N_{2}$ (Definition 10). Because $N$ is any an element of $\Phi, \Phi$ is the CPset of $N_{1}$ and $N_{2}$. 
According to Algorithm 1, there may be many core processes for two labeled WF-nets, and there is only sequential structure in each CP. Meanwhile, Algorithm 1 can be easily extended to multiple labeled WF-nets. If core processes of multiple process variants are gained, then all of them are sound. The core processes derived from Algorithm 1 can preserve soundness in the following theorem.

Theorem 2 Let $N_{i}=\left(P_{i}, T_{i}, F_{i}, L_{i}\right)(1 \leq i \leq m)$ be labeled WF-nets of a business process and $N=(P, T, F, L)$ be a CP of $N_{i}$. Then $N$ is sound.

Proof. Since $N=(P, T, F, L)$ has an only occurrence sequence $\sigma=[i] t_{1} M_{1} t_{2} M_{2} t_{3} \ldots M_{m-}$ ${ }_{1} t_{m}[o]$, an only token moves from $i$ towards $o$ via each $t \in T$.

(1)Proper completion: when $o$ is marked, all other places are empty, i.e. $o \in M$ implies $M=[o]$.

(2)Option to complete: for any $M \in[N,[i]>$, the only token move toward to o, i.e. $[o] \in[N, M>$.

(3)No dead transitions: each $t$ is on the only path from $i$ to $o$, i.e. $M[t>$ and $t$ is not dead. Therefore, $N$ is sound.

In Figure 2, $F_{N_{1}}=\{A B C\}, \quad F_{N_{2}}=\{A D C\} \quad$ and $L C S\left(F_{N_{1}}, \quad F_{N_{2}}\right)=\{A C\}$. Since $\left(\tau_{\{B\}}\left(N_{1}\right),[i]\right) \sim\left(C P_{1},[i]\right)$ and $\left(\tau_{\{D\}}\left(N_{2}\right),[i]\right) \sim\left(C P_{1},[i]\right)$, then $N_{1} \leq C P_{1}$ and $N_{2} \leq C P_{1}$ (Definition 9). In Figure 3, $F_{N_{1}}=\{A B C\}, F_{N_{2}}=\{A B C, A D C\}$ and $L C S\left(F_{N_{1}}, F_{N_{3}}\right)=\{A B C\}$. Because of $\left(N_{1},[i]\right) \sim\left(C P_{2},[i]\right)$ and $\left(\partial_{\{D\}}\left(N_{3}\right),[i]\right) \sim\left(C P_{2},[i]\right), N_{1} \leq C P_{2}$ and $N_{3} \leq C P_{2}$ (Definition 9). $C P_{1}$ is the CP of $N_{1}, N_{2}$, and $C P_{2}$ is the CP of $N_{1}$ and $N_{3}$ (Theorem 1). Furthermore, from Theorem 2, $C P_{1}$ and $C P_{2}$ are sound.

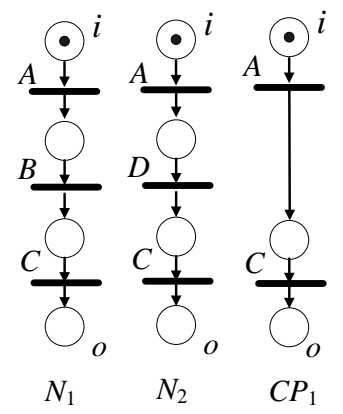

Figure 2. The CP of $N_{1}$ and $N_{2}$

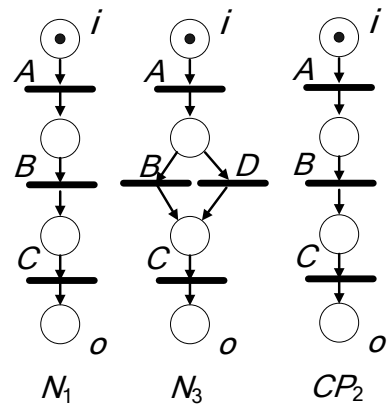

Figure 3. The CP of N1 and N3

\subsection{Business Process Adaptation}

After core processes describing the commonalities of process variants are found, a business process can be adjusted to adapt specific environment like specific law or 
company culture based on these core processes. The business process can be extended based on core processes, and extensional business processes are obtained.

Definition 13 (Extensional business process, EBP) Let $N_{1}=\left(P_{1}, T_{1}, F_{1}, L_{1}\right)$ be a labeled WF-net of a business process and $N_{2}=\left(P_{2}, T_{2}, F_{2}, L_{2}\right)$ be a CP of process variants. Let $N^{\prime}=\left(P^{\prime}, T^{\prime}, F^{\prime}, L\right)$ be a labeled WF-net such that $N^{\prime} \leq N_{1}$ and $N^{\prime} \leq N_{2}$. Then a labeled WF-net $E B P=(P, T, F, L)$ is an EBP of $N_{1}$ and $N_{2}$, and EBPset is the set of EBPs iff:

(1) For each $E B P \in E B P$ set, then $E B P \leq N_{1}$ and $E B P \leq N_{2}$; and

(2) For $N^{\prime}, \exists E B P \in E B P$ set such that $N^{\prime} \leq E B P$.

Note that an EBP is a subclass of $N_{1}$ and $N_{2}$. Furthermore, for any a subclass $N^{\prime}$ of $N_{1}$ and $N_{2}$, there is an EBP which is a superclass of $N^{\prime}$, i.e. each EBP is a minimal subclass of $N_{1}$ and $N_{2}$.

Some concepts are introduced to define EBP of labeled WF-nets in the following section.

Definition 14 (Pre-transition set and Post-transition set) Let $N=(P, T, F, L)$ be a labeled WF-net, then

${ }^{\circ} t=\left\{t^{\prime} \mid t^{\prime} \in T \wedge \exists p \in P:\left(t^{\prime}, p\right) \in F \wedge(p, t) \in F\right\}$ is called the pre-transition set of $t$;

$t^{\circ}=\left\{t^{\prime} \mid t^{\prime} \in T \wedge \exists p \in P:(t, p) \in F \wedge\left(p, t^{\prime}\right) \in F\right\}$ is called the post-transition set of $t$.

If $t_{i} \in{ }^{\circ} t_{i+1}(1 \leq i \leq n-1)$, then it is denoted as $t_{i} \in^{\circ+} t_{n}(i=1,2, \ldots, n-1)$.

If $t_{i} \in t_{i-1}{ }^{\circ}(2 \leq i \leq n)$, then it is denoted as $t_{i} \in t_{1}{ }^{\circ+}(2 \leq i \leq n)$.

Definition 15 (projection) Let $N=(P, T, F, L)$ be a labeled WF-net. Then $N^{\prime}=\left(P^{\prime}, T^{\prime}\right.$, $\left.F^{\prime}, L^{\prime}\right)$ is the projection of $N$ from $t_{i}$ to $t_{j}$, denoted $\Omega\left(N, t_{i}, t_{j}\right)$, where:

$\left.P^{\prime} \subseteq P ; T^{\prime}=\left\{t \mid t \in t_{i}{ }^{\circ+} \wedge t \in{ }^{\circ+} t_{j}\right)\right\}$

$F^{\prime}=F \cap\left(\left(T^{\prime} \times P^{\prime}\right) \cup\left(P^{\prime} \times T^{\prime}\right)\right)$; and

$L^{\prime}=\left\{(t, L(t)) \mid t \in T^{\prime}\right\}$.

Given a labeled WF-net $N=(P, T, F, L)$, one operation $\pi$ is defined to get the pre-set or post-set of transitions.

$\forall t \in T, \pi_{1}(t)=\{p \mid p \in P \wedge(p, t) \in F\} ; \pi_{2}(t)=\{p \mid p \in P \wedge(t, p) \in F\}$.

To gain an EBP, the mapping of nodes should be found between the business process and the core process, and labels of transitions can be used to establish the mapping of nodes. Given a labeled WF-net of a business process, the business process extension is the inverse of blocking and hiding operation according to the core processes. The construction algorithm can be described as follows.

\section{Algorithm 2 Construct EBPset}

Input: a labeled WF-net $N_{1}=\left(P_{1}, T_{1}, F_{1}, L_{1}\right)$ of a core process, a labeled WF-net $N_{2}=$ $\left(P_{2}, T_{2}, F_{2}, L_{2}\right)$ of a business process

\section{Output: $\Theta$}

\section{Begin}

S1: If $\exists t_{1} \in T_{1}$ and $t_{2} \in T_{2}$ such that $L_{1}\left(t_{1}\right)$ equal to $L_{2}\left(t_{2}\right)$, then match $t_{1}$ with $t_{2}$, meanwhile map $i_{1}, i_{2}$ to $i$ and map $o_{1}, o_{2}$ to $o$.

S2: If for $\forall t \in T_{1}, \exists t^{\prime} \in T_{2}$, such that $L_{1}(t)$ equal to $L_{2}\left(t^{\prime}\right)$, then $\Theta=\left\{N_{2}\right\}$, go to S4.

S3: For each $t \in T_{1}$ such that $L_{1}(t) \notin L_{2}$ do

3.1 For each $t^{\prime} \in{ }^{\circ} t$ \{While $\left.\left(L_{1}(t) \notin A_{2}\right) \quad\left\{t \vDash t^{\prime}\right\}, t_{11} \vDash t\right\}$.

3.2 For each $t^{\prime} \in{ }^{\circ} t$ \{While $\left.\left(L_{1}(t) \notin A_{2}\right)\left\{t \vDash t^{\circ}\right\}, t_{12} \vDash t\right\}$.

3.3 Let $N^{\prime}=\left(P^{\prime}, T^{\prime}, F^{\prime}, L^{\prime}\right)$ be $\Omega\left(N_{1}, t_{11}, t_{12}\right)$. Let $t_{21}, t_{22} \in T_{2}$ such that $L_{2}\left(t_{21}\right)$ equal to $L_{1}\left(t_{11}\right)$ and $L_{2}\left(t_{22}\right)$ equal to $L_{1}\left(t_{12}\right)$

3.4 If $t_{21} \in^{\circ} t_{22}$ then

For each $t_{21}, N=(P, T, F, L)$ where

$\left\{P=P_{2} \cup P^{\prime}-\left\{\pi_{2}\left(t_{21}\right)\right\}, T=T_{2} \cup T^{\prime}, L=L_{2} \cup L^{\prime}, F=F_{2} \cup F^{\prime} \cup\left\{t_{21} \times \pi_{2}\left(t_{11}\right), \pi_{1}\left(t_{12}\right) \times t_{22}\right)\right\}-$

$\left.\left\{t_{21} \times \pi_{2}\left(t_{21}\right), \pi_{2}\left(t_{21}\right) \times t_{22}\right\}\right\}$ 


\section{Endfor}

$$
\Theta=\Theta \cup\{N\}
$$

Else $N=(P, T, F, L)$ where

$$
\left\{P=P_{2} \cup P^{\prime}-\left\{\pi_{2}\left(t_{21}\right)\right\}, T=T_{2} \cup T^{\prime}, L=L_{2} \cup L^{\prime}, F=F_{2} \cup F^{\prime} \cup\left\{t_{21} \times \pi_{2}\left(t_{11}\right), \pi_{1}\left(t_{12}{ }^{\circ}\right) \times t_{21}{ }^{\circ}\right)\right\}-
$$

$\left.\left\{t_{21} \times \pi_{2}\left(t_{21}\right), \pi_{2}\left(t_{21}\right) \times t_{21}{ }^{\circ}\right\}\right\}$. or

$$
\left\{P=P_{2} \cup P^{\prime}-\left\{\pi_{1}\left(t_{22}\right)\right\}, T=T_{2} \cup T^{\prime}, L=L_{2} \cup L^{\prime}, F=F_{2} \cup F^{\prime} \cup\left\{{ }^{\circ} t_{22} \times \pi_{2}\left(t_{11}\right), \pi_{1}\left(t_{12}{ }^{\circ}\right) \times t_{22}\right)\right\}-
$$

$\left.\left\{{ }^{\circ} t_{22} \times \pi_{1}\left(t_{22}\right), \pi_{1}\left(t_{22}\right) \times t_{22}\right\}\right\}$. or

$$
\left\{P=P_{2} \cup P^{\prime}-\left\{\pi_{2}\left(t_{11}\right), \pi_{1}\left(t_{12}\right)\right\}, T=T_{2} \cup T^{\prime}, L=L_{2} \cup L^{\prime},\right.
$$

$\left.F=F_{2} \cup F^{\prime} \cup\left\{\pi_{2}\left(t_{21}\right) \times t_{11}{ }^{\circ},{ }^{\circ} t_{12} \times \pi_{1}\left(t_{22}\right)\right\}-\left\{\pi_{2}\left(t_{11}\right) \times t_{11}{ }^{\circ},{ }^{\circ} t_{12} \times \pi_{1}\left(t_{12}\right)\right\}\right\}$.

$\Theta=\Theta \cup N\}$

EndIF

EndFor

S4: Output $\Theta$.

End

\section{Figure 4. The EBPset Construction Algorithm}

For a $\mathrm{CP}$, there may be multiple EBPs on the base of a business process by Algorithm 2. In practice, which EBP will be chosen depends on the specific environment requirement. It is proved that $\Theta$ is the EBPset of $N_{1}$ and $N_{2}$ in the following conclusion.

Theorem 3 Let $N_{1}=\left(P_{1}, T_{1}, F_{1}, L_{1}\right)$ be a labeled WF-net representing a core process and $N_{2}=\left(P_{2}, T_{2}, F_{2}, L_{2}\right)$ be a labeled WF-net representing a business process. Let $N^{\prime}=\left(P^{\prime}\right.$, $T^{\prime}, F^{\prime}, L^{\prime}$ ) be a labeled WF-net such that $N^{\prime} \leq N_{1}$ and $N^{\prime} \leq N_{2}$. If $\Theta$ is obtained by Algorithm 2 , then $\forall N \in \Theta, N=(P, T, F, L)$ is an EBP of $N_{1}$ and $N_{2}$.

Proof. (1) From Algorithm 2, $N$ has a source place $i$ such that ${ }^{\circ} i=\varnothing$ and a sink place $o$ such that $o^{\bullet}=\varnothing$. Each node of $N$ is on a directed path from $i$ to $o$, i.e. $N$ is strongly connected. So, $N$ is a labeled WF-net.

(2)For $\forall N \in \Theta, \quad$ let $\quad A_{0}=A_{1}-A_{2}, \quad I=\left\{L(t) \mid t \in\left(\left(^{\circ+} t\right)^{\circ+} \wedge L(t) \in A_{0}\right\} \quad\right.$ and $H=A_{0}-I$, then $\left(\tau_{l^{\circ}} \partial_{H}(N),[i] \sim\left(N_{1},[i]\right)\right.$. i.e. $N \leq N_{1}$.

$N \leq N_{2}$ can be proved similarly.

(3)Let $\quad N^{\prime} \leq N_{1}$ and $N^{\prime} \leq N_{2}$, denoted $\exists I_{1}, \quad H_{1}, \quad I_{2} \quad$ and $H_{2} \in L^{\prime}$, such that $\left(\tau_{I 1}{ }^{\circ} \partial_{H_{1}}\left(N^{\prime}\right),[i]\right) \sim\left(N_{1},[i]\right)$ and $\left(\tau_{I_{2}}{ }^{\circ} \partial_{H_{2}}\left(N^{\prime}\right),[i]\right) \sim\left(N_{2},[i]\right)$ then $\exists N=(P, T, F, L) \in \Theta$, such that $\left(\tau_{I \cap \cap I^{\circ}} \partial_{H 1 \cap H_{2}}\left(N^{\prime}\right),[i]\right) \sim(N,[i])$, i.e. $N^{\prime} \leq N$. $N_{2}$.

So, $N$ is an EBP of $N_{1}$ and $N_{2}$ by Definition 13. Furthermore, $\Theta$ is the EBPset of $N_{1}$ and

Theorem 4 shows that an EBP obtained by Algorithm 2 preserves soundness.

Theorem 4 Let $N_{1}=\left(P_{1}, T_{1}, F_{1}, L_{1}\right)$ be a labeled WF-net representing a core process and $N_{2}=\left(P_{2}, T_{2}, F_{2}, L_{2}\right)$ be a labeled WF-net representing a business process. Let $N=(P$, $T, F, L)$ be an EBP from $N_{1}$ and $N_{2}$ by Algorithm 2. If $N_{1}$ and $N_{2}$ are sound, then $N$ is sound.

Proof. Let $L^{\prime}=A_{1}-A_{2}$, then $\exists I$ and $H \subseteq L^{\prime}$, such that $\left(\tau_{I^{\circ}} \partial_{H}(N),[i]\right) \sim\left(N_{2},[i]\right)$ (Theorem 3). For $t(L(t) \in I),(t, L(t))$ is added to $N_{2}$ in sequence pattern. For $t^{\prime}\left(L\left(t^{\prime}\right) \in H\right),\left(t^{\prime}, L\left(t^{\prime}\right)\right)$ is added to $N_{2}$ in xor-split/xor-join pattern. Then an EBP $N$ is obtained by Algorithm 2. All these operations don't influence the soundness of labeled WF-nets. If $N$ is not sound, then $N_{1}$ and/or $N_{2}$ are not sound. It contradicts with the hypothesis, so $N$ is sound.

A CP and an EBP can preserve the soundness of original labeled WF-nets. So, proposed algorithms are effective and feasible.

\section{Application Instance}


Many business processes exist to provide business solutions in typical functional areas. And these processes are typically individualized for countries and enterprises [3]. When these enterprises enter other markets, original business processes are often not suitable for the new market rule or local laws. Some work should be carried to acquire suitable business processes for new environments. Tax declaring is taken an example to show the business process adaption due to the extensively applied for enterprises. Figure 5 and Figure 6 respectively describe the declaring tax processes of enterprises in region $R_{1}$ and $R_{2}$ (all these processes have been simplified). In Figure 5, the process of enterprise $\mathrm{E}_{1}\left(N_{4}\right)$ is made up of tax_appl (tax application), asse_tax (self assessment of taxation), VAT_start (VAT start), pay_VAT (pay valued-added tax), VAT_comp (VAT completion), and tax_comp (tax completion), and the process of enterprise $\mathrm{E}_{2}\left(N_{5}\right)$ includes input_tax (input tax) and output_tax (output tax) instead of pay_VAT in $N_{4}$. In Figure 6, the process of enterprise $\mathrm{E}_{3}\left(N_{6}\right)$ includes pay_res_tax (pay resource tax) and pay_VAT_add_tax (pay VAT additional tax) except the activities in $N_{1}$ and $N_{2}$. The process of enterprise $\mathrm{E}_{4}\left(N_{7}\right)$ includes pay_pro_tax (pay property tax) other than pay_res_tax in $N_{6}$.

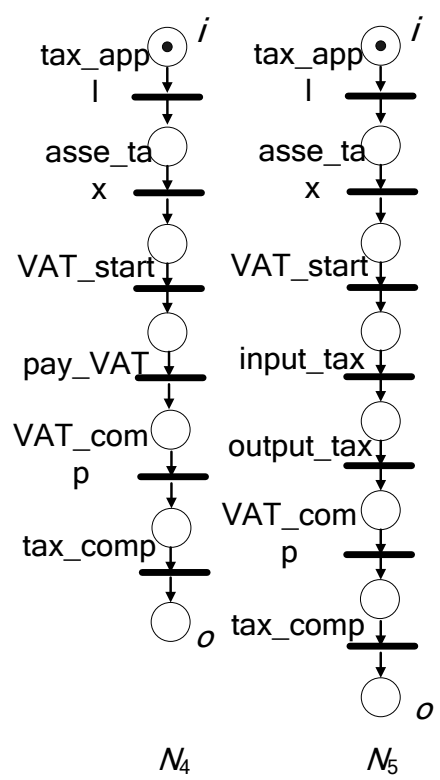

Figure 5. Two Declare Tax Processes in $R_{1}$ 


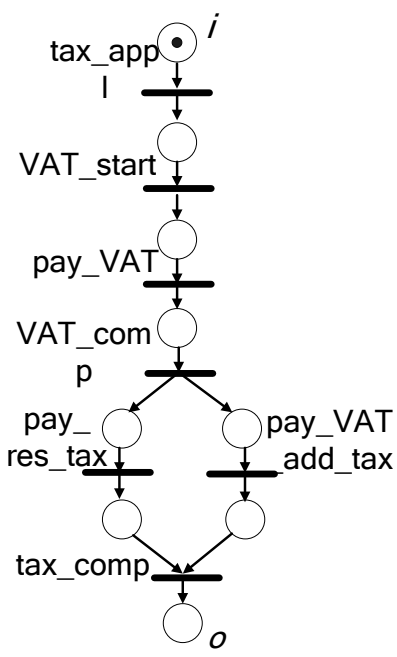

$N_{6}$

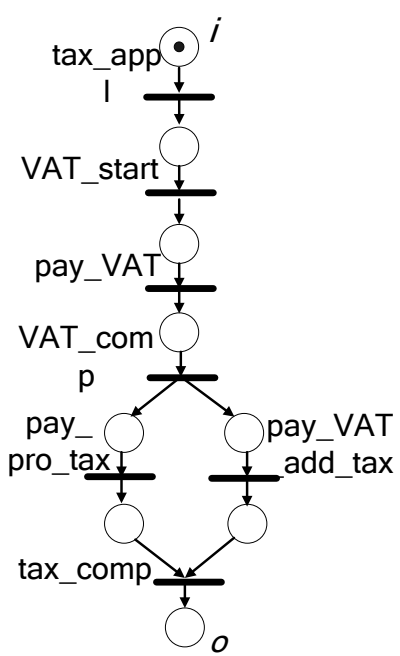

$N_{7}$

Figure 6. Two Declare Tax Processes in R2

According to the method in [8], the models that share common fragment can be used to construct union of models (called merged models). Models $N_{M}$ and $N_{M^{\prime}}$ in Figure 7 describe merged models of declare tax processes in $R_{1}$ and $R_{2}$ respectively. Merged models can represent and replace original models of the same underlying process. But, when enterprises $E_{2}$ of $R_{1}$ enter the market of $R_{2}$, existing declare tax processes, such as $N_{2}$, and merge model $N_{M}$ is not suitable for $R_{2}$ because there are extra taxes in declare tax process for $R_{2}$. While $N_{6}, N_{7}$ and $N_{M}$, is appropriate for $R_{2}$, they can not describe this situation well due to the activity asse_tax of $N_{5}$ is ignored. Similarly, configurable processes in [3] can not represent the processes adaption across regions although its functions and connectors are configurable.
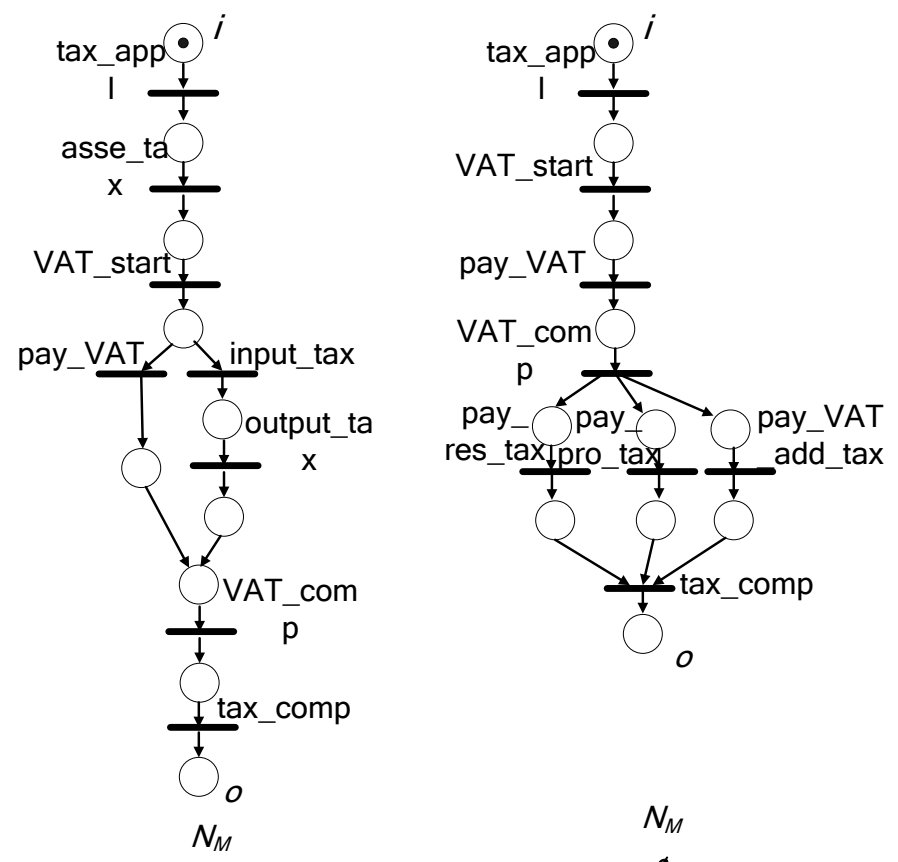

Figure 7. Merged Processes of Declare Tax Processes in R1 and R2 
To adapt the process $N_{5}$ to meet the requirement of $R_{2}$, the core process $N_{C P}$ of $N_{6}$ and $N_{7}$ can be got according to Algorithm 1. In Figure 8, $N_{C P}$ represents the commonalities of declare tax process in region $R_{2}$. Theorems 1 and 2 can be combined to show that $N_{C P}$ is a $\mathrm{CP}$ of processes $N_{6}$ and $N_{7}$, and $N_{C P}$ is sound.

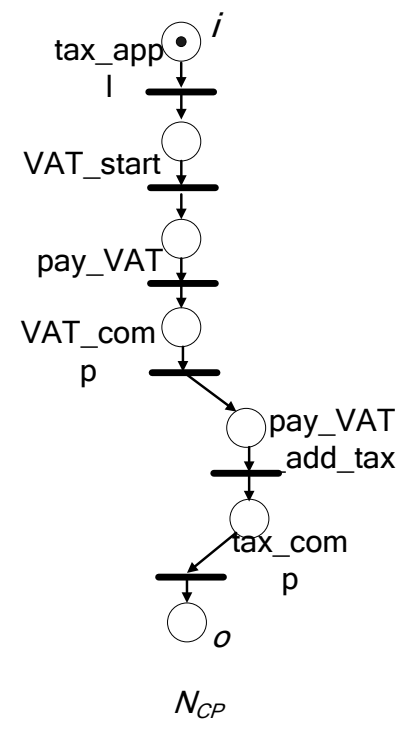

Figure 8. The CP of $N_{6}$ and $N_{7}$

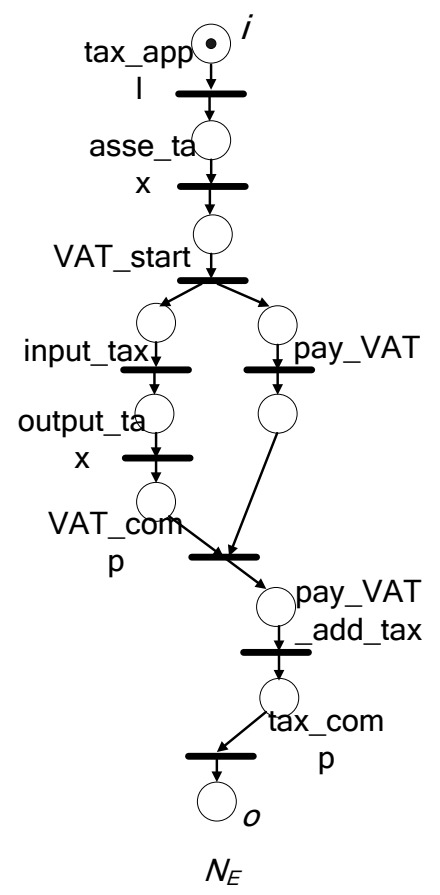

Figure 9. The EBP NE of N5 and NCP

In Figure 9, EBP $N_{E}$ is acquired based on declare tax processes $N_{5}$ and $N_{C P}$ according to Algorithm 2. The activity pay_VAT of $N_{C P}$ is similar to activities input_tax and output_tax of $N_{2}$, and they are contained to $N_{E}$ in a xor-split/xor-join pattern. Meanwhile, $N_{E}$ contains the pay_VAT_add_tax of $N_{C P}$ and asse_tax of $N_{5}$. Thus, $N_{E}$ reserves the characteristic of $N_{5}$ and embodies the commonalities of process in region $R_{2}$. Compared 
with original process $N_{5}$ and merged models $N_{M}$ and $N_{M}, N_{E}$ is more suitable to the specific environment of region $R_{2}$. By Theorem 3 and Theorem $4, N_{E}$ is sound due to the soundness of $N_{5}$ and $N_{C P}$.

\section{Related Works}

Business process management is progressively paid attention to in current research work. And it often occurs that multiple variants of a business process exist, and these variants usually originated from different companies or units. How to manage and use these variants is an important part of business process management. Merged model [8] is proposed to converge process variants into a single process. Meanwhile, the unions of process variants (called merged model) subsume a collection of process models and intersections of process variants (called digests) identify the recurring fragments. Although the commonalities and differences between multiple variants are found, all these ones are applied to replace variants with consolidated model, and process adaption to other environments is not involved.

In some papers, relevant business processes is constructed as a repository (called reference models) which accelerate the modeling process [3, 9]. For example, configurable EPC [3] provides related constraint information of functions and connectors on reference models, and these information make model user can individualise the model by selecting alternative options on a type level. Within each organization, an individual process can be got according to constraint information. Despite diversity of one process among organizations, a process variant can not be applied directly to other regions because the reference model itself is typically individual for countries or enterprises. The commonalities of variant are involved in [6-7]. Common Divisor (MCD) of object life cycle [6] describes the common behavior of objects by hiding and blocking operations and it provides the basis of this paper. But the characteristic and property of MCD are not involved. In [7], core process, the common segment of process variants, is defined from the spectrum of structural and behavioral aspects. Neither algorithm nor further application of the core process is discussed. In this paper, core processes are introduced to adapt the existing process to other regions. And core processes embody the maximal commonalities of the processes in new region. By extending the existing process in the base of core processes, new processes are suitable for the new region reserving the characteristic of existing process. And related theorems prove that core processes and extensional processes are sound.

\section{Conclusions}

A business process is modelled as a labeled WF-net based on labeled P/T-nets, and the inheritance relation between labeled WF-nets is proposed based on branching bisimilarity and behavioral equivalence. A core process is proposed to describe the commonalities of process variants, and related algorithms are designed and theorems are proved. Existing process model can be extended based on core processes, then extensional business processes are acquired. Extensional business processes has the characteristic of original process model and core processes, so it is suitable to the environment of process variants. The existing methods put emphasis on management of process variants in one region, and extensional business processes are exclusively designed for cross regions in this paper. When an enterprise expands its market to a new region, original processes need adapt themselves to the new economical conditions. We get the new economical rules by researching process variants in the new region and then extend original processes to adapt the new rules. And Extensional business processes can fit the new economical condition well. 


\section{Acknowledgements}

This work is supported by the National Basic Research Program of China under grant 2010CB328101; the National Natural Science Foundation of China under grants 61170078 and 61173042; the Doctoral Program of Higher Education of the Specialized Research Fund of China under grant 20113718110004; Basic Research Program of Qingdao City of China under grant 131-4-116-jch; and the SDUST Research Fund of China under Grant 2011KYTD102.

\section{References}

\subsection{Journal Article}

[2] C. Li, M. Reichert, and A. Wombacher. "The MinAdept Clustering Approach for Discovering Reference Process Models Out of Process Variants". International Journal of Cooperative Information systems, no. 19 (2010), pp. 3-4.

[3] M. Rosemann, W.M.P. van der Aalst. A configurable reference modelling language. Information Systems. vol. 1, no. 32 (2007).

[4] W.M.P. van der Aalst. "Business process management: A comprehensive survey". ISRN Software Engineering, 1, 20131 (2013)

[5] W.M.P. van der Aalst, M. Dumas, and F. Gottschalk, A. H. M. ter Hofstede, M.La.Rosa, J. Mendling. "Preserving correctness during business process model configuration", Formal Aspects of Computing, no. 22 (2010), pp. 3-4.

[7] M. Weidlich, M. Weske. "Structural and behavioural commonalities of process variants". in ZEUS, (2010).

[8] M. La Rosa, M. Dumas, and R. Uba, R. Dijkman. "Business process model merging: an approach to business process consolidation". ACM Transactions on Software Engineering and Methodology (TOSEM), vol. 2, no. 22 (2013).

[9] F. Gottschalk, W.M.P. van der Aalst, M.H. Jansen-Vullers, H. Monque, and M. La Rosa. "Configurable workflow models". International Journal of Cooperative Information Systems, vol. 2, no. 17 (2008).

[10] H.M.W. Verbeek, T. Basten, W.M.P. van der Aalst. "Diagnosing workflow processes using Woflan", The Computer Journal, vol. 4, no. 44 (2001).

[11] W.M.P. Van der Aalst. "The application of Petri nets to workflow management. Journal of circuits", systems, and computers. vol. 01, no. 8 (1998).

[12] Y.Y. Du, C.J. Jiang, M.C. Zhou, "A Petri net-based model for verification of obligations and accountability in cooperative systems", IEEE T Syst Man Cy A, vol. 39, no. 2, (2009).

[13] Q. Hu, Y.Y. Du, S.X. Yu, "Service net algebra based on logic Petri nets", J Inf Sci, no. 268,(2014).

[14] YY Du, LQi, MC Zhou. "A vector matching method for analysing logic Petri nets". Enterprise Information System.vol. 4, no. 5 (2011).

[16] R.J. Van Glabbeek, W.P. Weijland, "Branching time and abstraction in bisimulation semantics", Journal of the ACM (JACM), vol. 3, no. 43, (1996).

\subsection{Book}

[1] M. Weske, Editor, "Business Process Management: Concepts, Languages, Architectures, SpringerVerlag", Berlin (2007).

\subsection{Conference Proceedings}

[6] W.M.P. van der Aalst, T. Basten, "Identifying commonalities and differences in object life cycles using behavioral inheritance", in: J.M. Colom and M. Koutny(Eds.), Application and Theory of Petri Nets 2001, volume 2075 of Lecture Notes in Computer Science, (2001) Springer-Verlag, Berlin.

[15] J. Desel, W. Reisig. Place/transition Petri nets, in W. Reisig and G. Rozenberg(Eds), Lectures on Petri Nets I: Basic Models, volume 1491 of Lecture Notes in Computer Science, (1998) Springer-Verlag, Berlin. 


\section{Authors}

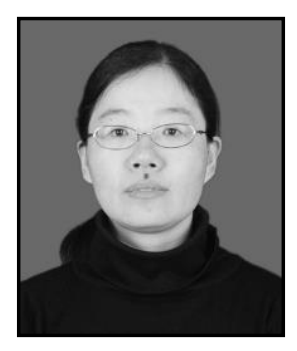

Hongxia Li is a Ph.D. student in School of Information Science and Engineering at Shandong University of Science and technology, China. She received her Master degree in computer science and technology from Shandong University in 2004. Her research focuses on Petri net, process mining and workflow theory.

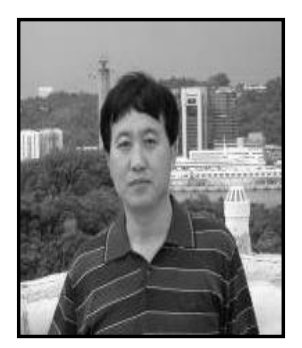

YuYue Du received the B.S. degree from Shandong University, Jinan, China, in 1982, the M.S. degree from Nanjing University of Aeronautics and Astronautics, Nanjing, China, in 1991, and the Ph.D. degree in computer application from Tongji University, Shanghai, China, in 2003. He is currently a Professor at the College of Information Science and Engineering, Shandong University of Science and Technology, Qingdao, China. He is a member of Professional Committee of Petri Nets of China Computer Federation. His research interests are in formal engineering, Petri nets, real-time systems, Web services, and workflows. He has taken in over ten projects supported by National Nature Science Foundation, National Key Basic Research Developing Program and other important and key projects at provincial levels. He has published more than 140 papers in domestic and international academic publications, and they are embodied more than 80 times by SCI and EI, cited more than 270 times by others. 